\title{
Novos desafios da reabilitação em pacientes DPOC
}

\author{
New challenges for rehabilitation in DPOC patients \\ Nuevos retos para la rehabilitación en pacientes con DPOC
}

Recebido: 14/03/2021 | Revisado: 20/03/2021 | Aceito: 24/03/2021 | Publicado: 01/04/2021

\author{
Bruna Pereira Nagamine \\ ORCID: https://orcid.org/0000-0002-8799-2529 \\ Faculdade Guaraí, Brasil \\ E-mail: brunapnagamine@gmail.com \\ Daniela Maristane Vieira Lopes Maciel \\ ORCID: https://orcid.org/0000-0001-5934-0219 \\ Faculdade ITOP Palmas, Brasil \\ E-mail: danielafisiomaciel@gmail.com
}

\begin{abstract}
Resumo
A doença pulmonar obstrutiva crônica (DPOC) é a campeã em causas de mortalidade e morbidade no mundo, caracterizada por uma desordem progressiva que leva a falência respiratória devido uma obstrução do fluxo aéreo expiratório, acompanhada de hipersecreção de muco em diferentes graus e a perda progressiva da função pulmonar. $\mathrm{O}$ objetivo deste artigo é demonstrar a importância da reabilitação pulmonar em pacientes com DPOC. O estudo é uma revisão de literatura de caráter qualitativo e descrititvo, pesquisado em bases se dados como Public Medlineor Publisher Medline (PubMed), Cochrane Library, Scientific Eletronic Library Online (SciELO) e Sociedade Brasileira de Pneumologia e Tisiologia, com os descritores , "Doença Obstrutiva", "Hipersecreção", "Fisioterapia". Os déficits fisiológicos da DPOC incluem a restrição da tolerância ao exercício físico, limitação do fluxo aéreo, hiperinsuflação pulmonar, fraqueza dos músculos respiratórios, limitação ventilatória e consequentes prejuízos na qualidade de vida. A reabilitação pulmonar vem neste contexto promover um recondicionamento cardiorrespiratório, auxiliar na desobstrução brônquica e equalização dos fluxos respiratórios, promover ganhos na mobilidade torácica e fortalecimento dos músculos respiratórios, aumento da capacidade funcional, maior tolerância ao exercício, melhorando da sensação de dispnéia e da fadiga. Percebe-se que a reabilitação pulmonar é uma ferramenta indispensável e coadjuvante dentro do processo de melhora clínica do paciente com DPOC, há ganhos positivos no que tange a qualidade de vida, condicionamento cardiorrespiratório e queda dos episódios de agudização dos sintomas e eventuais hospitalizações.
\end{abstract}

Palavras-chave: Doença obstrutiva; Hipersecreção pulmonar; Fisioterapia.

\begin{abstract}
Abstrat
Chronic obstructive pulmonary disease (COPD) is the champion in causes of mortality and morbidity in the world, characterized by a progressive disorder that leads to respiratory failure due to an obstruction of the expiratory airflow, accompanied by hypersecretion of mucus in different degrees and progressive loss lung function. The aim of this article is to demonstrate the importance of pulmonary rehabilitation in patients with COPD. The study is a literature review of a quantitative and descriptive character, researched in databases such as Public Medlineor Publisher Medline (PubMed), Cochrane Library, Scientific Eletronic Library Online (SciELO) and Brazilian Society of Pulmonology and Tisiology, with the descriptors, " Obstructive Disease "'," Hypersecretion "," Physiotherapy ". Physiological deficits in COPD include restricted exercise tolerance, limited airflow, pulmonary hyperinflation, respiratory muscle weakness, ventilatory limitation and consequent impairments in quality of life. In this context, pulmonary rehabilitation promotes cardiopulmonary reconditioning, aids in bronchial clearance and equalization of respiratory flows, promotes gains in chest mobility and strengthening of respiratory muscles, increased functional capacity, greater tolerance to exercise, improving the feeling of dyspnea and fatigue It is noticed that pulmonary rehabilitation is an indispensable and supporting tool within the process of clinical improvement of patients with COPD, there are positive gains with regard to quality of life, cardiorespiratory conditioning and a decrease in episodes of acute symptoms and eventual hospitalizations.
\end{abstract}

keywords: Obstructive disease; Pulmonary hypersecretion; Physiotherapy.

\section{Resumen}

La enfermedad pulmonar obstructiva crónica (EPOC) es la campeona en causas de mortalidad y morbilidad en el mundo, caracterizada por un trastorno progresivo que conduce a insuficiencia respiratoria por obstrucción del flujo de aire espiratorio, acompañado de hipersecreción de moco en diferentes grados y pérdida progresiva. función pulmonar. El objetivo de este artículo es demostrar la importancia de la rehabilitación pulmonar en pacientes con EPOC. El estudio es una revisión de la literatura de carácter cualitativo y descriptivo, investigada en bases de datos como Public Medline 
o Publisher Medline (PubMed), Cochrane Library, Scientific Eletronic Library Online (SciELO) y Sociedad Brasileña de Neumología y Tisiología, con los descriptores, "Obstructive Enfermedad "," Hipersecreción "," Fisioterapia ". Los déficits fisiológicos en la EPOC incluyen tolerancia restringida al ejercicio, flujo de aire limitado, hiperinsuflación pulmonar, debilidad de los músculos respiratorios, limitación ventilatoria y consecuentes deficiencias en la calidad de vida. En este contexto, la rehabilitación pulmonar promueve el reacondicionamiento cardiopulmonar, ayuda en el aclaramiento bronquial y la igualación de los flujos respiratorios, promueve ganancias en la movilidad del tórax y fortalecimiento de los músculos respiratorios, aumento de la capacidad funcional, mayor tolerancia al ejercicio, mejorando la sensación de disnea y fatiga Se advierte que la rehabilitación pulmonar es un herramienta indispensable y de apoyo dentro del proceso de mejoría clínica de los pacientes con EPOC, se obtienen ganancias positivas en términos de calidad de vida, acondicionamiento cardiorrespiratorio y disminución de episodios de síntomas agudos y eventuales hospitalizaciones.

Palabras clave: Enfermedad obstructiva; Hipersecreción pulmonar; Fisioterapia.

\section{Introdução}

A doença pulmonar obstrutiva crônica (DPOC) é a principal causadora de morbidade e mortalidade no mundo, com prevalência crescente caracterizada por uma obstrução do fluxo aéreo expiratório que apresenta hipersecreção de muco em diferentes graus e a perda progressiva da função pulmonar, associada a efeitos sistêmicos como as disfunções musculoesquelticas e inflamação das vias aéreas. É definida por uma desorganização progressiva da mecânica pulmonar levando a falência respiratória, sendo a segunda patologia mais comum dentre os segurados da Previdência Social (Global, 2017; Cassio, 2019).

A diminuição do fluxo aéreo ou obstrução brônquica é a principal característica da patologia, que ocorre devido á inflamação das vias aéreas, destruindo o parênquima pulmonar e favorecendo o aprisionamento do ar, desequilibrando tanto a função quanto a estrutura pulmonar (Sousa et al., 2011).

Os déficits fisiológicos da patologia incluem a restrição da tolerância ao exercício, que leva a limitação das atividades de vida diária (AVDS), a limitação do fluxo aéreo e a hiperinsuflação, consequentemente fraqueza dos músculos inspiratórios, além do individuo passar a respirar em volumes pulmonares mais altos se aproximando da capacidade pulmonar total, o que proporciona uma limitação ventilatória (Ribeiro et. al., 2005). Essa interação das anormalidades dos músculos ventilatórios, periféricos e cardiovasculares exemplifica a intolerância ao realizar exercícios físicos e até durante a realização das atividades de vida diária (AVDs) e atividades simples refletindo em prejuízos na qualidade de vida (Guenette; Webb; O’donnell, 2012).

A reabilitação pulmonar auxilia no recondicionamento do sistema cardiorrespiratório, na desobstrução brônquica, na correção das deformidades posturais, melhorando a ventilação mecânica, aumentando a capacidade funcional, reduzindo o número de hospitalizações e melhorando a capacidade para a realização de exercício, melhorando com isso a sensação de dispnéia, os níveis de disfunções emocionais e a fadiga, sendo o fisioterapeuta um profissional insdispensável nesse contexto. Diante disso, o objetivo desse estudo é evidenciar a importância da reabilitação pulmonar em pacientes com DPOC (Gava; Picanço, 2007).

\section{Metodologia}

Este estudo trata-se de uma revisão bibliográfica, de caracteristica descritiva e exploratória, com abordagens qualitativa. Tendo como princípio para sua discussão teórica, artigos científicos (Pereira et al., 2018). Foram selecionados 59 artigos e excluídos 35 por inconformidade. Todos os textos examinados foram criteriosamente avaliados por dois autores independentes, sendo excluídos aqueles materiais com baixa qualidade metodológica, texto incompleto que abordavam sobre a reabilitação pulmonar em pacientes com DPOC.

O levantamento de dados foi realizado atraves de pesquisas em bancos de dados e guidessline nacionais e internacionais como Public Medlineor Publisher Medline (PubMed), Cochrane Library, Scientific Eletronic Library Online (SciELO), Sociedade Brasileira de Pneumologia e Tisiologia, American Thoracic Society, Congressos e livros, entre janeiro e novembro 
de 2020. Foram utilizados os descritores na língua portuguesa como Doença Obstrutiva, Hipersecreção Pulmonar, Fisioterapia e seus correlatos na língua inglesa Obstructive Disease, Pulmonary Hypersecretion, Physiotherapy.

\section{Resultados e Discussão}

\section{Alterações funcionais em pacientes com DPOC}

Nos indivíduos com DPOC as deficiências fisiológicas incluem a limitação do fluxo aéreo, dispnéia, hiperinsuflação, exacerbação aguda caracterizada pela piora da dispnéia, tosse e produção de escarro, tornando-se os principais motivos de hospitalizações e morte em pacientes DPOCíticos e a exacerbação resulta em um decaimento acelerado da função pulmonar, dessa maneira, é importante a intervenção fisioterapêutica na reabilitação pulmonar (Celli; Macnee, 2014).

A fraqueza nos músculos inspiratórios influenciam na redução do desempenho ao exercício e na dispnéia. O principal motivo dessa fraqueza é a hiperinsuflação pulmonar que reduz a cápsula do diafragma ocasionando mudanças geométricas nos músculos intercostais, fazendo com que os mesmos trabalhem de forma ineficiente, ocorrendo o aumento do volume pulmonar no final da expiração, reduzindo a capacidade inspiratória, fazendo com que chegue perto da capacidade pulmonar total (Dourado et al., 2006).

Indivíduos com DPOC apresentam hipertrofia das células que são responsáveis pela produção de muco, aumentando a secreção de muco nas vias aéreas, provocando a obstrução e a resistência das vias aéreas, limitando o fluxo aéreo, aumentando o volume residual e diminuindo a eficácia do diafragma, e a primeira alteração apresentada e a musculoesquelética, que compromete os músculos respiratórios e periféricos, que reduzem a atividade enzimática aeróbica, aumenta a apoptose, abaixa a fração de fibras musculares do tipo I, reduz a capilaridade e essas alterações reduzem a capacidade aeróbica, ocorrendo acidose lática precoce, e o metabolismo anaeróbico que facilita no aumento da demanda ventilatória, além de fraqueza muscular em pacientes com doença pulmonar obstrutiva crônica que é proveniente da inflamação sistêmica que se encontra no período de exacerbação, desequilíbrio nutricional, estresse oxidativo, descondicionamento, distúrbios eletrolíticos, falência cardíaca, redução do anabolismo, hipoxemia, corticosteróides sistêmicos e hipercapnia (Paulin; Brunetto; Carvalho, 2013).

Desordens psiquiátricas também podem acometer o paciente com DPOC sendo as mais comuns ansiedade e depressão, que podem ser marcadas por redução do sono, aumento da letargia, pessimismo, dificuldade em concentração, isolamento social e redução do apetite, sendo associada ao comprometimento das atividades funcionais, redução dos hábitos de higiene e a dificuldade em controlar a exacerbação da patologia (Carneiro et al., 2010).

\section{DPOC e Hiperinsuflação Pulmonar}

Segundo o Jornal Brasileiro de Pneumologia, Cordoni et al., o principal atributo fisiológico da DPOC é a limitação crônica do fluxo aéreo provocado pela obstrução das vias aéreas e destruição irreversível do parênquima pulmonar. A ação inflamatória crônica que se encontra presente em pacientes de DPOC ocasiona alterações estruturais que diminui o diâmetro das vias aéreas. Já a eliminação do parênquima pulmonar leva ao decréscimo da elasticidade e ocasiona danos nas estruturas vasculares adjacentes. A desarmonia entre as forças de recolhimento e expansão pulmonar restringe a capacidade das vias aéreas de se manterem abertas, de maneira que permita que o volume final da expiração se torne maior, levando ao aprisionamento aéreo, consequentemente, a hiperinsuflação pulmonar.

E o surgimento da hiperinsuflação dinâmica (HD) no tempo da realização do exercício é prevalente em pacientes portadores de DPOC, desde aqueles com acometimento grave, moderada e leve. A hiperinsuflação dinâmica é o resultado de uma restrição do fluxo respiratório diante de um tempo expiratório que é diminuído ao esforço, em função do aumento da demanda ventilatória. Nos portadores de DPOC que possuem uma restrinção do fluxo expiratório mesmo em repouso, a 
hiperinsuflação dinâmica, é retrato da mudança da capacidade residual funcional (CRF) que vai em direção à CPT, sendo a única tática que permite um aumento de fluxo na expiração e, conseqüentemente, provocando um aumento no volume corrente (O’donnell; Webb, 2008).

A força muscular inspiratória ocasionada permite uma boa resposta no crescimento do volume, que com o decréscimo das demandas de ventilação essa é uma estratégia tolerada. No entanto, para se utilizar de ventilações mais altas (esforços inten sos e moderados), a CRF move-se cada vez mais em direção à CPT, e a sua força inspiratória é incapaz de ocasionar um crescimento proporcional de volume (dissociação neuromecânica), estando, em uma porção menos favorável do sistema respiratório. Dessa maneira, conseqüentemente, a dispnéia aumenta gradativamente, e o paciente com DPOC anula o esforço. Além da sobrecarga ventilatória, a hiperinsuflação dinâmica concede a determinação dos efeitos adversos sobre o sistema cardiovascular e a musculatura inspiratória, possibilitando a redução do débito cardíaco. E a hiperinsuflação pulmonar estática (HPE) pode acontecer devido à diminuição do recolhimento elástico pulmonar, restrição do fluxo expiratório ou ambos, ocasionando o aprisionamento aéreo (Maccari, 2015).

A HPE tem como principal característica o aumento da capacidade residual funcional (CRF) que resulta na soma do volume de reserva expiratório (VRE) e do volume residual (VR). Dessa maneira, as medidas da CRF e do VR são relevantes durante a avaliação da HPE, por ambos refletirem no volume de ar remanescente nos pulmões, em conseqüente, o aprisionamento aéreo. A HPE permite a limitação da capacidade de aumentar a ventilação em resposta às condições metabólicas no desempenho de exercícios (O’donnell; Webb, 2008).

Em função às reabilitações terapêuticas, a diminuição da HD diante do esforço é associada a um decréscimo da dispnéia, como a utilização de ventilação com pressão positiva, broncodilatadores de longa e curta duração, suplementação de oxigênio e de mistura hélio-oxigênio, e reabilitação que atua na diminuição da resistência das vias aéreas e na demanda ventilatória (Maccari, 2015).

\section{Reabilitação Cardiopulmonar}

Adaptado ao tratamento individualizado do paciente, a reabilitação é indicada para melhorar o estado funcional, reduzir os sintomas, amplificar a adesão do tratamento e reverter ás manifestações da doença. A reabilitação pulmonar melhora a suplementação de oxigênio, a dispnéia, o estado de saúde, a capacidade cardiopulmonar. Pacientes com doença pulmonar obstrutiva crônica tendem a adotar um estilo de vida sedentário, ocasionado pela fadiga ao exercício e a dispnéia. O descondicionamento físico consecutivo e as alterações nas funções e estruturas dos músculos periféricos resultam em mais fadiga e dispnéia, impulsionando ao paciente ser mais sedentário. A reabilitação cardiopulmonar aumenta a capacidade de promover a atividade física e o exercício (Gava; Picanço, 2007).

A reabilitação ajuda a reduzir a demanda ventilatória, reduzindo a frequiência respiratória, com um tempo expiratório maior permitindo uma menor hiperinsuflação dinâmica reduzindo a dispnéia e é relacionada á melhora psicológica, expandindo o desempenho cognitivo e diminuindo a depressão e a ansiedade. Os exercícios aumentam o envio de neurotransmissores cerebrais, como dopamina, serotonina, norepinefrina e endorfinas, reduzindo a depressão, além de, reduzir a citocina, aumentar o fluxo sanguíneo cerebral, a atividade metabólica cerebral, o transporte e utilização do oxigênio cérebro. Além disso, a reabilitação pulmonar pode abordar a perda de peso, perda de massa muscular, e o fraco condicionamento físico e os exercícios respiratórios são relevantes no programa de reabilitação pulmonar com a finalidade de reduzir a hiperinsuflação dinâmica, reduzindo a dispnéia, promovendo a melhora da troca gasosa e o aumento da força dos músculos respiratórios (Machado, 2008).

As técnicas respiratórias mais comuns para diminuir a dispnéia incluem o frenolabial onde se aplica uma resistência expiratória variável, contraindo os lábios aumentando o tempo expiratório e a respiração diafragmática que consiste em respirações profundas e lentas sem efeitos na ventilação minuto, a melhora da função dos músculos inspiratórios esta associada 
com o desempenho ao exercício, que pode ser evidenciado pelo teste de caminhada de seis minutos, e na sensação de dispnéia durante a realização das AVDs, e os exercícios aeróbicos são um grande aliado para os pacientes com DPOC, aumentando a concentração de enzimas oxidativas mitocondriais, a capilarização dos músculos envolvidos, o limiar anaeróbio, o $\mathrm{VO}_{2}$ máx e a diminuição do tempo de recuperação da creatina fosfato $(\mathrm{CP})$, resultando na melhora da capacidade de realização do exercício. Um dos principais benefícios do condicionamento aeróbico na reabilitação pulmonar é o aumento da distancia percorrida no teste de caminhada de seis minutos ( Alves; Santos; Melo, 2007; Rubin, 2008).

O método pilates criado por Joseph Pilates contribui na reabilitação desses pacientes fortalecendo o core, que tem como função alongar, fortalecer e melhorar a funcionalidade respiratoria (Ellsworth, 2012), a sua estabilização melhora a resistencia e potencia muscular da lombar, pélve e quadril e aumenta a força muscular (Hoogenboom; Bennett, 2015). Um dos intuitos dos exercicios do método é fortalecer os musculos do abdome, ganhar força muscular, e alongar a muscultura, e esses exercicios favorecem o condicionamento fisico, o cordiorespiratorio, o realinhameto corporal que facilita na realização das atividades de vida diaria e esses exercicios são compostos por contrações isotonicas e isometricas (Navega et al., 2016).

Os exercícios resistidos tem se mostrado eficaz por promover a melhora das disfunções musculoesqueleticas provocadas pela DPOC, além de previner doenças adjacentes e o sedentarismo. Esses exercícios tem como principal característica estimular a formação da massa óssea, além de, promover a qualidade de vida, diminuir os fatores de risco por lesões como torções, risco de quedas, desaceleração ou aceleração brusca, e o treinamento resistido utilizando peso aumenta a força muscular, melhora a capacidade funcional (Farias; Rodrigues, 2009). Suetta et al. (2004) comprovam que o treinamento resistido, proporciona o aumento da massa muscular, o aumento da força muscular máxima e dos estímulos neurais.

As manobras de higiene brônquica auxiliam na mobilização e na diminuição das secreções pulmonares, favorecendo a limpeza das vias aéreas respiratórias, melhorando as trocas gasosas. As manobras tem como vontagem propiciar o deslocamento e a remoção das secreções o que exige uma menor alteração na pressão pleural e a probabilidade de colapso das vias aéreas é menor (Gambaroto, 2006).

O threshold (aparelho com carga linear pressórica) tem sido considerado um recurso de grande eficácia no treinamento muscular respiratorio, pois exerce uma função importante na reabilitação de pacientes com DPOC por promover a reeducação funcional respiratoria, criando uma maior resistencia a quadros de dispneia, aumentando a tolerancia ao exercicio e melhorando a força muscular inspiratória por propiciar carga inspiratoria constante e sem alterações da pressão (Britto; Brant; Parreira, 2009).

O European Respiratory Journal desenvolveu um estudo onde vinte pacientes foram treinados utilizando o threshold e os resultdos obtidos foram satisfatorio por promoverem o alívio da dispneia, melhora do condicionamento fisico e trabalho respiratorio, diminuindo o volume-minuto e o consumo de oxigênio.

A respiração com pressão positiva intermitente (RPPI) é uma forma de exercicio respiratorio que se utiliza a pressão positiva para aumentar o volume pulmonar e a capacidade vital, que auxilia na expansão pulmonar (Carvalho, 2009). Handelsman demonstra que a utilização da RPPI é eficiente ao ser utilizada na exarcebação dos sintomas da DPOC, e esta relcionada com a melhora do quadro clinico desses pacientes.

A expansão pulmonar que o RPPI proporciona faz com que aumente a pressão intrapulmonar, esse aparelho aumenta a pressão de abertura das vias aereas que vai ocasionar na diferença de pressão necessaria para ocorrer o fluxo inspiratório (Guimarãaes; Menezes; Oliveira, 2009). O RPPI tem como objetivo melhorar os parâmetro de oxigenação, aumentar a capacidade inspiratória, volume corrente, favorecendo a eliminiação de secreção e a tosse.

\section{Conclusão}

A adoção de um programa de reabilitação cardiopulmonar conduzido pelo fisioterapeuta para indivíduos com DPOC é salutar e resultarão em um melhor funcionamento da mecânica respiratória, equalização das pressões respiratórias, fortalecimento 
muscular, tolerância ao exercício, independência funcional no desempenho das AVD's, melhora da sensação de fadiga, mudanças na qualidade de vida e consequentemente, diminuição das manifestações clínicas, das necessidades médicas e hospitalares.

O tema abordado no presente artigo é de relevância cientifica por se tratar de um assunto muito comum e recorrente e é de grande importância novas pesquisas para verificar a eficiência de novos recursos para otimizar a reabilitação cardiopulmonar em pacientes com DPOC.

\section{Referências}

Alves, C. E., Santos, J. G., \& Melo, P. L. (2007). Otimização do uso do Flutter VRP1 em Reabilitação Respiratória: Caracterização Mecânica e Desenvolvimento de Programas de Apoio ao Usuário. IFMBE Proceedings 18:1020-3, CLAIB 2007.

Britto, R. R., brant, T. C. \& parreira, V. F. (2019). Recursos Manuais e Instrumentais em Fisioterapia Respiratória. Manole, 2009. Cad. Bras. Ter. Ocup., 27(1), 27-34.

Carneiro, et al. (2010). Risco de reiternamento na doença pulmonar obstrutiva crônica- estudo prospectivo com ênfase no valor da avaliação da qualidade de vida e depressão. Revista portuguesa de pneumologia 2010.

Carvalho, L. C. \& Pessoa, S. R.. (2009). Epidemiologia da DPOC nos presentes aspectos nacionais. Rev. Pulmão Rj. Autorizações temáticas

Dourado V. Z., \& Godoy, I. (2004). Recondicionamento muscular na DPOC: principais intervenções e novas tendências. Rev Bras Med Esporte.

Ellsworth, A. (2012) Treinamento do Core: Anatomina Ilustrada: Gui Completo Para o Fortalecimento Do Core. Tradução de Marcos Ikeda. Manoli.164 p

Farias, I. S. R., \& Rodrigues, T. S. (2009). Exercício Resistido - Na saúde, na doença, no envelhecimento.

Gambaroto \& Gilberto. (2006). Fisioterapia Respiratória em Unidade de Terapaia Intensiva. Atheneu

Gava, M. V., Picanço, P. S. A. (2006). Fisioterapia Pneumológica. Manole.

Global initiative for chronic obstructive lung disease - GOLD. (2017). Global Strategy for the Diagnosis, management, and prevention of Chronic Obstructive Pulmonary Disease updated Fontana. USA: GOLD, 2017.

Guenette, J. A., Webb, K. A., \& O’Donnell, D. E. (2012). Does dynamic hyperinflation contribute to dyspnoea during exercise in patients with COPD? Eur Resp J 40:322-29.

Handelsman, H. Intermittent positive pressure breathing (IPPB) therapy. Health Tchnol Assess Rep, 1:1-9.

Hoogenboom, B. J., \& Bennett, J. L. (2015). Técnicas de Exercícios Terapêuticos: Estratégias de Intervenção Musculoesquelética. Tradução de Juliana Cristina Frare. Manoli, 333-410

J Bras Pneumol. (2015),41(5):467-472 http://dx.doi.org/10.1590/S1806-37132015000000035

Machado MGR. Reabilitacao Pulmonar. (2008). In: Bases da Fisioterapia Respiratoria - Terapia Intensiva e Reabilitação. Editora Guanabara Koogan, 2008.

National Institute for Health and Clinical Excellence. (2004). Chronic obstructive pulmonary disease: management of chronic pulmonary obstructive disease in adults in primary and secondary care (partial update). London, National Clinical Guideline Centre.

Navega, M. T. et al.(2016). Efeitos do Método Pilates solo no equilíbrio e na hipercifose torácica em idosas: Ensaio clinico controlado randomizado. Rev. Bras. Geriatr. Gerontol. 19(3), 465-472, 2016.

O’Donnell, D. E., \& Webb, K. A. (2008). The major limitation to exercise performance in COPD is dynamic hyperinflation. J Appl Physiol.

Pereira A. S. et al. (2018). Metodologia da pesquisa científica. UFSM.

Rubin, A. S. et al. (2008). Resposta broncodilatadora imediata ao formoterol em doença pulmonar obstrutiva crônica com pouca reversibilidade. J. bras. pneumol., 34(6), 373-379.

Sociedade Brasileira de Pneumologia e Tisiologia (SBPT) (2002). II Consenso Brasileiro sobre Espirometria. J Pneumol, 28(3):S1-S82.

Suetta, C., Aagaard, P., \& Rosted, A. (2004). Training-induced changes in muscle CSA, muscle strength, EMG and rate of force development in elderly subjects after long-term unilateral disuse. J Appl Physiol 2004, 97:1954-61 Sociedade Brasileira de Pneumologia e Tisiologia. II Consenso Brasileiro de Doença Pulmonar obstrutiva crônica (DPOC). J Bras Pneumol, 30(5):1-42.

Wrobel, J. P., Thompson, B. R., Stuart-Andrews, C. R., Kee, K., Snell, G. I., Buckland, M., \& Williams, T. J. (2015). Intermittent positive pressure ventilation increases diastolic pulmonary arterial pressure in advanced COPD. Heart Lung, 44(1):50-6 